\title{
Antioxidant Enzymes and their Role in Preventing Cell Damage
}

\author{
Amra Bratovcic* \\ Department of Physical Chemistry and Electrochemistry, University of Tuzla, \\ Bosnia and Herzegovina \\ *Corresponding Author: Amra Bratovcic, Department of Physical Chemistry \\ and Electrochemistry, University of Tuzla, Bosnia and Herzegovina.
}

Received: February 20, 2020

Published: February 29, 2020

(C) All rights are reserved by Amra

Bratovcic.

DOI: $10.31080 /$ ASNH.2020.04.0659

\begin{abstract}
Reactive oxygen species (ROS), such as superoxide anion $\left(\mathrm{O}_{2}{ }^{--}\right)$, nitric oxide (NO•) hydrogen peroxide $\left(\mathrm{H}_{2} \mathrm{O}_{2}\right)$, and hydroxyl radical (HO•), consist of radical and non-radical oxygen species formed by the partial reduction of oxygen. The accumulation of ROS in cells may cause damage of nucleic acids, proteins, lipids and may cause cell death and trigger oxidative stress which yield to the development and progression of several diseases. Furthermore, ROS may promote tumour metastasis through gene activation. It is important to emphasize that equilibrium between the production and elimination of toxic levels of ROS is sustained by enzymatic and nonenzymatic antioxidants. When oxidative stress arises as a consequence of high level of ROS, a defence system promotes the regulation and expression of several nonenzymatic and enzymatic antioxidant. To cope with potentially damaging ROS, aerobic tissues contain endogenously produced antioxidant enzymes such as superoxide dismutase (SOD), glutathione peroxidase (GPx), and catalase and several exogenously acquired radical-scavenging substances such as vitamins E and C, carotenoids and tocopherols. Afterward, both zinc and selenium are intimately involved in protecting the body against oxidant stress. In addition, it was reveal that supplementation with exogenous antioxidants or boosting of endogenous antioxidants is a promising method of countering the undesirable effects of oxidative stress on the human body.
\end{abstract}

Keywords: Reactive Oxygen Species; Antioxidant Enzymes; Cell Damage

\section{Abbreviations}

Reactive Oxygen Species (ROS); Superoxide Dismutase (SOD); Glutathione Peroxidase (GPx); Reactive Nitrogen Species (RNS)

\section{Introduction}

Oxidative stress (OS) is a cellular phenomenon or condition which occurs as a result of physiological imbalance between the levels of antioxidants and oxidants (free radicals or reactive species) in favour of oxidants. In other words, oxidative/nitrosative stress is the result of disequilibrium in oxidant/antioxidant which reveals from continuous increase of reactive oxygen species (ROS) and reactive nitrogen species (RNS) production [1].

ROS is a collective term used for a group of oxidants, which are either free radicals or molecular species capable of generating free radicals [2]. These free radicals, which can be found as oxygen derived (ROS) or nitrogen derived (RNS) have rather high reactivity and short life. Generally, ROS/RNS are generated as by-products of cellular metabolism and ionizing radiation. Therefore, different reactive species are involved in cellular oxidative stress and oxidative damage. The common name for these reactive species is "free radicals". Free radicals are defined as "any chemical species capable of independent existence that contains one or more unpaired electrons".
Components that are present as free radicals in ROS are superoxide, (02-•), hydroxyl, (HO•), hydroperoxyl, $\left(\mathrm{HO}_{2}\right)$, peroxyl, (RO0•), and alkoxyl, (RO•), while those nonradicals refer to hydrogen peroxide, $\left(\mathrm{H}_{2} \mathrm{O}_{2}\right)$, hypochlorous acid, $(\mathrm{HClO})$, ozone $\left(\mathrm{O}_{3}\right)$, and singlet oxygen, $\left(\mathrm{1O}_{2}\right)$. Meanwhile, nitric oxide ( $\mathrm{NO} \bullet$ ), nitrogen dioxide $\left(\mathrm{NO}_{2}\right)$, dinitrogen trioxide, $\left(\mathrm{N}_{2} \mathrm{O}_{3}\right)$, and peroxynitrite, (ONOO-) are the free radicals derived from RNS $[3,4]$.

The three primary species, i.e. the superoxide anion $\left(\mathrm{O}_{2}-^{\bullet}\right)$, hydrogen peroxide $\left(\mathrm{H}_{2} \mathrm{O}_{2}\right)$ and the hydroxyl radical $(\mathrm{HO} \bullet)$ are called ROS because they are oxygen-containing compounds with reactive properties. $\mathrm{O}_{2}-\bullet$ and $\mathrm{HO} \bullet$ are commonly referred to as "free radicals". They can react with organic substrates and lead to intermediate species able to further produce other ROS. Superoxide anion is produced by the addition of a single electron to oxygen, and several mechanisms exist by which superoxide can be produced in vivo. For instance, $\mathrm{H}$ atom abstraction by $\mathrm{HO} \bullet$ free radicals on a $\mathrm{C}-\mathrm{H}$ bond leads to a carbon-centered radical, that further reacts rapidly with $\mathrm{O}_{2}$ to give a peroxyl radical $\mathrm{RO}_{2} \bullet[5]$.

The latter may react with another substrate to give a new carbon-centered radical and a hydroperoxide ROOH, which may decompose into alkoxyl radical RO• in a reaction catalyzed by redox competent metal cations such as iron or copper, as occurring with 
heme proteins [6]. These "secondary" species are all ROS and share a similarity in structure and reactivity with the three primary species $\mathrm{O}_{2} \bullet-, \mathrm{H}_{2} \mathrm{O}_{2}$ and $\mathrm{HO} \bullet$. Among them, $\mathrm{H}_{2} \mathrm{O}_{2}$ (and hydroperoxides) is a molecular species and is supposed to be less reactive than the other radical short-lived species that are able to react with a range of targets (an exception may apply for 02•-). However, its toxicity can be exerted via Fenton reaction in the presence of redox metal ions such as iron or copper, or via Haber-Weiss reaction in the presence of $\mathrm{O}_{2} \bullet-[7]$.

Transition metals like iron and copper play a key role in the production of hydroxyl radicals in vivo. Hydrogen peroxide reacts with iron II (or copper I) to generate the hydroxyl radical, a reaction first described by Fenton.

$\mathrm{Fe}^{2+}+\mathrm{H}_{2} \mathrm{O}_{2} \rightarrow \mathrm{Fe}^{3+}+\mathrm{OH}^{\cdot}+\mathrm{OH}^{-}$

This reaction occur in vivo, but the situation is complexed by the fact that superoxide anion (the main source of hydrogen peroxide in vivo) normally also be present [8].

Superoxide anion and hydrogen peroxide react together directly to produce the hydroxyl radical, but the rate constant for this reaction in aqueous solution is actually zero. However, if transition metal ions are present a reaction sequence is established that can proceed at a rapid rate:

$\mathrm{Fe}^{3+}+\mathrm{O}_{2-} \rightarrow \mathrm{Fe}^{2+}+\mathrm{O}_{2}$

$\mathrm{Fe}^{2+}+\mathrm{H}_{2} \mathrm{O}_{2} \rightarrow \mathrm{Fe}^{3+}+\mathrm{OH}^{\cdot}+\mathrm{OH}^{-}$

net result

$\mathrm{O}_{2^{-}}+\mathrm{H}_{2} \mathrm{O}_{2} \rightarrow \mathrm{OH}^{-}+\mathrm{OH}^{\bullet}+\mathrm{O}_{2}$

The net result of the reaction series illustrated above is known as the Haber-Weiss reaction.

Most of the oxygen taken up by the cells is converted to water by the action of cellular enzymes. However, some of these enzymes leak electron into oxygen molecules and lead to the formation of free radicals. They are also formed during normal biochemical reactions involving oxygen. ROS are produced from molecular oxygen, during the successive 4 steps of 1-electron reduction. Free radicals are claimed to be harmful to humans because its unpaired electron(s) extracts electron(s) from other molecules in the body to gain stability, hence damaging DNA, proteins, and lipids [9].

The other biologically important free radicals exist such as lipid hydroperoxide (ROOH), lipid peroxyl radical (ROO), and lipid alkoxyl radical (RO), which are associated with membrane lipids, then nitric oxide (NO), nitrogen dioxide $\left(\mathrm{NO}_{2}\right)$ and peroxynitrite (ONOO-), which are reactive nitrogen species and thiol radical (RS), which has an unpaired electron on the sulfur atom $[10,11]$.

Superoxide anion $\left(\mathrm{O}_{2}^{-}\right)$is produced by the addition of a single electron to oxygen, and several mechanisms exist by which superoxide can be produced in vivo [12]. Any biological system gener- ating superoxide anion also forms hydrogen peroxide $\left(\mathrm{H}_{2} \mathrm{O}_{2}\right)$ as a result of a spontaneous dismutation reaction. In addition, some enzymatic reactions may produce hydrogen peroxide directly [13] which itself is not a free radical as it does not contain any unpaired electrons. However, it is a precursor to certain radical species such as peroxyl radical, hydroxyl radical, and superoxide.

Oxidative stress appears to be the foundation for the induction of multiple cellular pathways associated with damage of important biomolecules and subcellular structures in cells.

The hydroxyl radical $(\bullet \mathrm{OH})$ is probably the final mediator of most free radical induced tissue damage [14]. All of the ROS described above exert most of their pathological effects by giving rise to hydroxyl radical formation. The reason for this is that the hydroxyl radical reacts, with extremely high rate constants, with almost every type of molecule found in living cells such as lipids and nucleotides. Although hydroxyl radical formation can occur in several ways, by far the most important mechanism in vivo is likely to be the transition metal catalysed decomposition of superoxide anion and hydrogen peroxide [15].

In Figure is shown the influence of excess of reactive oxygen species (ROS) and reactive nitrogen species (RNS) and their impact on human health.

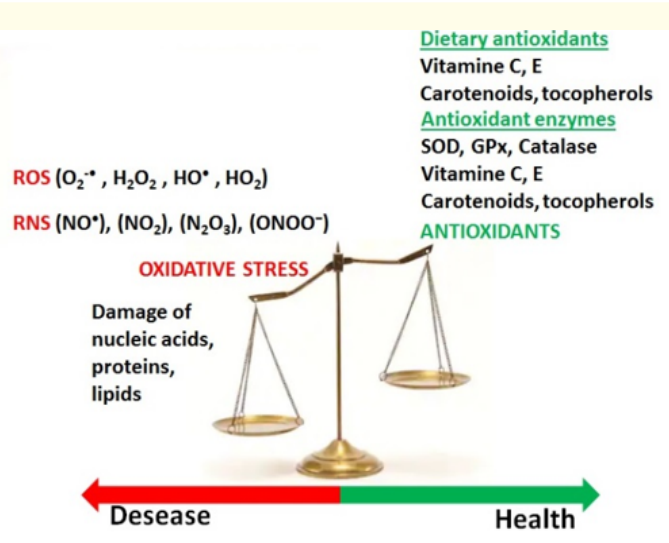

Figure 1: Schematic illustration of the influence of excess of reactive oxygen species (ROS) and reactive nitrogen species (RNS) on human health.

Some people confuse antioxidants with antioxidant enzymes. Antioxidants help repair damage done by free radicals in the body and the resulting oxidation. Enzymes, however, attempt to stop damage before it occurs by triggering chemical reactions that rid the body of free radicals and dangerous oxygen in the form of oxides.

In the first part of this paper oxidative stress and reactive oxygen species are well defined and explained in detail. Then, antioxidant enzymes, as well as vitamines, carotenoides and tocopherols and food antioxidants. The aim of this paper is to highlight the importance of the antioxidant enzymes in prevention of cell damage. 


\section{Antioxidant enzymes}

The human body has several mechanisms to counteract oxidative/nitrosative stress by producing antioxidants. Haida and Hakiman [16] have reported a comprehensive review related to antioxidants which may be grouped into enzymatic and nonenzymatic antioxidants. Antioxidants have gained attention at the global scale on its prominent beneficial roles that can fight against many chronic infirmities, including cancer [2] and cardiovascular diseases. They have reported that most studies had looked into nonenzymatic antioxidants due to lack of references on enzymatic antioxidant assays. Therefore, that review article depicts on seven assays of enzymatic antioxidants (superoxide dismutase, catalase, peroxidase, ascorbate peroxidase, ascorbate oxidase, guaiacol peroxidase, and glutathione reductase) and fifteen activities of nonenzymatic antioxidants (total polyphenol, total phenolic acids, total flavonoids, total ascorbic acid, anthocyanin content, DPPH scavenging activity, FRAP assay, hydrogen peroxide scavenging activity, nitric oxide scavenging activity, superoxide radical scavenging activity, hydroxyl radical scavenging activity, phosphomolybdate assay, reducing power, metal ion chelating activity, and $\beta$-carotene), which are described in detail to ease further investigations on antioxidants in future.

Patients suffering from inflammatory diseases often present with diminished levels of antioxidants either due to insufficient dietary intake or even more likely, due to increased demand in situations of overwhelming ROS production by activated immune effector cells like macrophages.

During cellular immune response, interferon $\gamma$-dependent pathways are activated such as tryptophan breakdown by the enzyme indoleamine 2,3-dioxygenase (IDO) in monocyte-derived macrophages, fibroblasts, endothelial and epithelial cells. Neopterin, a marker of oxidative stress and immune activation is produced by GTP-cyclohydrolase I in macrophages and dendritic cells. Nitric oxide synthase (NOS) is induced in several cell types to generate nitric oxide (NO). NO, despite its low reactivity, is a potent antioxidant involved in the regulation of the vasomotor tone and of immunomodulatory signalling pathways. NO inhibits the expression and function of IDO. Function of NOS requires the cofactor tetrahydrobiopterin (BH4), which is produced in humans primarily by fibroblasts and endothelial cells. Highly toxic peroxynitrite (ONOO-) is formed solely in the presence of superoxide anion $\left(\mathrm{O}_{2}\right.$ ). Neopterin and kynurenine to tryptophan ratio (Kyn/Trp), as an estimate of IDO enzyme activity, are robust markers of immune activation in vitro and in vivo. Both these diagnostic parameters are able to predict cardiovascular and overall mortality in patients at risk. Likewise, a significant association exists between increase of neopterin concentrations and Kyn/Trp ratio values and the lowering of plasma levels of vitamin-C, -E and -B. Vitamin-B deficiency is usually accompanied by increased plasma homocysteine [17].

In 2018, Ighodaro and Akinloye [18] reported that antioxidants such as polyphenols, ascorbic acid, vitamin A, alpha-lipoic acid, thioredoxin, glutathione, melatonin, coenzyme $\mathrm{Q}$, beta carot- enoids, alpha-tocopherols as well as antioxidant enzymes including superoxide dismutase, catalase, glutathione peroxidases, glutathione reductases and glutathione transferases have been widely investigated for the prevention and treatment of diseases resulting from oxidative damage. They highlight that the role and effectiveness of superoxide dismutase (SOD), catalase (CAT) and glutathione peroxidase (GPx) is important and indispensable in the entire defence strategy of antioxidants, especially in reference to super oxide anion radical $\left(\bullet \mathrm{O}_{2}\right)$ which is perpetually generated in normal body metabolism, particularly through the mitochondrial energy production pathway (MEPP).

\section{Superoxide dismutase (SOD)}

Superoxide dismutases (SODs) are a group of metalloenzymes that are found in all kingdoms of life. Superoxide dismutases (SODs) constitute a very important antioxidant defense against oxidative stress in the body. The enzyme acts as a good therapeutic agent against reactive oxygen species-mediated diseases. However, the enzyme has certain limitations in clinical applications. Therefore, SOD conjugates and mimetics have been developed to increase its therapeutic efficiency [19].

The enzyme can serve as an anti-inflammatory agent and can also prevent precancerous cell changes [20]. Natural SOD levels in the body drop as the body ages [21] and hence as one age, one becomes more prone to oxidative stress-related diseases. SOD mimetics are synthetic compounds that mimic the native SOD by effectively converting $\mathrm{O}_{2}$ - into $\mathrm{H}_{2} \mathrm{O}_{2}$, which is further converted into water by catalase. They are of prime interest in therapeutic treatment of oxidative stress because of their smaller size, longer half-life, and similarity in function to the native enzyme.

\section{Glutathione peroxidase (GPx)}

Glutathione peroxidase is an antioxidant enzyme class with the capacity to scavenge free radicals. This is in turn helps to prevent lipid peroxidation and maintain intracellular homeostasis as well as redox balances [22].

Catalase is an antioxidant enzyme present in all aerobic organisms. It is known to catalyse $\mathrm{H}_{2} \mathrm{O}_{2}$ into water and oxygen in an energy-efficient manner in the cells exposed to environmental stress [23].

\section{Vitamins, carotenoids and tocopherols}

Today, most people are constantly exposed to stress, and as a result, various health problems with frequent cancer diagnoses are reported and as a result they are increasingly turning to the use of natural remedies since ancient times. In fact, many plants contain the necessary nutritional properties, minerals and vitamines necessary for the normal growth and development of healthy cells within the body and have a positive health effect [24]. Vitamins, minerals, amino acids, fatty acids and some carbohydrates that provide energy are essential nutrients [25].

Vitamins E consists of four tocopherols: $\alpha, \beta, \gamma$ and $\delta$, and the corresponding tocotrienols: $\alpha-, \beta, \gamma$ and $\delta$, which contain unsatu- 
rated side chains. The $\alpha$-tocopherol is the most biologically active form. Most plant-derived foods, especially fruits and vegetables, contain low-to-moderate levels of vitamin E activity; however, due to the abundance of plant-derived foods in our diets, they provide a significant and consistent source of vitamin E [26]. Vitamin E ( $\alpha$-tocopherol) functions as an essential lipid soluble antioxidant, scavenging hydroperoxyl radicals in lipid milieu. Vitamin E, a potent peroxyl radical scavenger, is a chain-breaking antioxidant that prevents the propagation of free radicals in membranes and in plasma lipoproteins [27]. When peroxyl radicals (RO0•) are formed, these react 1000-times faster with vitamin E (Vit E-OH) than with polyunsaturated fatty acids (PUFA) [28]. The hydroxyl group of tocopherol reacts with the peroxyl radical to form the corresponding lipid hydroperoxide and the tocopheryl radical (Vit E-0•). The tocopheryl radical (Vit E-0•) reacts with vitamin C (or other hydrogen donors, $\mathrm{AH}$ ), thereby oxidizing the latter and returning vitamin $\mathrm{E}$ to its reduced state [29]. The interaction of vitamins $E$ and $C$ has led to the idea of "vitamin E recycling", where the antioxidant function of oxidized vitamin $\mathrm{E}$ is continuously restored by other antioxidants.

Vitamin C (ascorbic acid) is an essential cofactor for $\alpha$-ketoglutarate-dependent dioxygenases. The health-promoting effects of vitamin $\mathrm{C}$ can be attributed to its biological functions as a co-factor for a number of enzymes, most notably hydroxylases involved in collagen synthesis, and as a water-soluble antioxidant. Examples are prolyl hydroxylases, which play a role in the biosynthesis of collagen and in down-regulation of the hypoxia-inducible factor (HIF) a transcription factor that regulates many genes responsible for tumour growth, energy metabolism, and neutrophil function and apoptosis.

As an antioxidant, vitamin C provides protection against oxidative stress-induced cellular damage by scavenging of reactive oxygen species [30].

\section{Carotenoids and tocopherols}

Carotenoids and tocopherols are powerful antioxidants synthesized in plants from a common precursor. Carotenoids are terpenoid-based compounds produced by most plants and a variety of bacteria and fungi. Carotenoids are mostly recognized for their vitamin A activity, as some can be cleaved in vivo via beta-carotene oxygenase 1 (BCO1) into vitamin A active compounds. In addition, carotenoids have shown to act, at least in vitro, as antioxidants, with a high potential to quench liposoluble radicals, as well as singlet oxygen [31].

\section{Food antioxidants}

Antioxidants, natural or synthetic food preservatives, are additives that preserve food from "farm to plate" and militate against oxidative deterioration on storage and processing. Due to their high stability and low volatility, the antioxidants help to maintain the level of nutrients, the texture, colour, taste, freshness, functionality, aroma, and appeal to consumers such as the older person, ceteris paribus. Antioxidants [32] are not only in food additives but are also to be found in food supplements and levels should be measured, as such, in body tissues and fluids [33]. Lesser known sources of antioxidants to that cited in reference [30] abound, for example, black chokeberry (Aronia melanocarpa) found in juices, purees, jams, and so forth which, containing high levels of polyphenols and flavonoids, has potential interventive value for a range of chronic diseases such as diabetes and cardiovascular diseases [34]. Fresh orange juice is considered as one of the healthiest beverages because of its wide range of health benefits, which include its ability to boost immunity, reduce signs of aging, prevent cancer, boost cellular repair and metabolism, detoxify the body, improve circulation and blood pressure, reduce inflammation and lower cholesterol levels [35]. Fermented grain food supplements also contain antioxidants, e.g., antioxidant biofactor, reducing lipid oxidation by scavenging upon the peroxyl radical [36]. Food antioxidants are scavengers of "free" (an unnecessary term) radicals, which is a chemical structure that has at least one unpaired electron which can cause cellular and genetic changes due to their highly reactive state that can act to produce damage over the nm range, e.g., the hydroxyl (HO•) radical; other oxygen radicals include the hydroperoxyl ( $\mathrm{HOO} \bullet$ ), alkyloxyl $(\mathrm{ROO} \bullet)$, and superoxide anion $\left(\mathrm{O}_{2}-\bullet\right)$; an important nitrogen containing radical is nitric oxide ( $\mathrm{NO} \bullet$ ); sulphur containing radicals include thiols (RS•) and disulphide anions (RSSR-•) and carbon containing radicals include the carbonate $\left(\mathrm{CO}_{3}^{-\bullet}\right)$ group [37].

\section{Supplementation with exogenous antioxidants}

It is believed that two-thirds of the world's plant species have medicinal importance, and almost all of these have excellent antioxidant potential. The interest in the exogenous plant antioxidants was first evoked by the discovery and subsequent isolation of ascorbic acid from plants. Nowadays, it is commonly accepted that diets that are high in fruits and vegetables protect against several human diseases, some of which are especially serious such as cardiovascular diseases and cancer. Several existing studies indicate that protective effects may result from intake of the antioxidants that are present in fruit and vegetables. Many natural compounds have been considered, either singularly or in combination, for supplementation therapies. Among them, particular attention was devoted to a specific subset of molecules such as vitamin C, vitamin E, resveratrol, curcumin, hydroxytyrosol and coenzyme Q10 [38].

Ascorbic acid is the main form of vitamin $\mathrm{C}$ in the human body and acts as the co-substrate for several enzymes. Its antioxidant activity relies on the ability to be reversibly oxidized to ascorbyl radical and then to dehydroascorbate (DHA) [39].

When referring to vitamin $\mathrm{E}$, a family of 8 isoforms classified in two categories is considered: four saturated analogues $(\alpha, \beta, \gamma$, and $\delta$ ) called tocopherols and four unsaturated analogues indicated as tocotrienols, which differ for the methylation pattern. These molecules are hydrophobic fat-soluble compounds found in a variety of food sources such as corn oil, peanuts, vegetable oils, fruits and vegetables [40]. 
Resveratrol (3, 4', 5-trihydroxystilbene) is a phytoalexin that belongs to the stilbene class of compounds, abundant in many plants, such as peanuts, blueberries, pine nuts and grapes where it mainly accumulates in a glycosylated form, and that is synthesized in response to fungal infection and to some environmental stresses like climate, ozone and ultraviolet irradiation [41].

Curcumin is a lipophilic bioactive phenol derived from the rhizome of Curcuma longa, which shows low solubility and stability in aqueous solution. It is contained in culinary curry and used as a colouring agent in food. Extensive research during the last few decades has suggested a strong therapeutic and pharmacological potential of this molecule as antioxidant, antimutagenic, antiprotozoal and antibacterial agent [42].

Curcumin strong medicinal properties are also associated with reported anti-cancer and neuroprotective effect such as in $\mathrm{Al}$ zheimer disease [43].

Hydroxytyrosol is an ortho-diphenol (a catechol) abundant in olive, fruits and extra virgin olive oil. This compound, due to its catecholic structure, shows a marked antioxidant activity and is able to scavenge oxygen and nitrogen free radicals, inhibit LDL oxidation, platelet aggregation and endothelial cell activation, and protects DNA from oxidative damage $[44,45]$.

Hydroxytyrosol is also a metal chelator and is able to scavenge the peroxyl radicals and break peroxidative chain reactions producing very stable resonance structures [46].

Coenzyme Q10 (CoQ10), referred to as ubiquinol in its most active (95\%) and reduced form (Q10H2), is a lipophilic molecule present in the membranes of almost all human tissues, and essential for the respiratory transport chain. CoQ10 is also capable of recycling and regenerating other antioxidants such as $\alpha$-tocopherol and ascorbate. CoQ10 has also been identified as a modulator of gene expression and inflammatory processes [47].

The quinol prevents lipid peroxidation by inhibiting the initial formation and propagation of lipid peroxy radicals, and in the process it is oxidized to the quinone and $\mathrm{H}_{2} \mathrm{O}_{2}$ is produced. In addition, it has been shown to protect proteins from oxidation by a similar mechanism [48], and to prevent oxidative DNA damage such as strand breakages. CoQ is also believed to function in the blood to protect lipoproteins such as very low density (VLDL), low density (LDL) and high density (HDL) lipoproteins from oxidation [49].

\section{Conclusions}

Reactive oxygen species (ROS) and reactive nitrogen species (RNS) are generated as by-products of cellular metabolism and ionizing radiation. This paper explains the mechanism of ROS and RNS formation and their detrimental effect on human health if present in greater quantities than antioxidants. In addition, the difference between antioxidants and antioxidant enzymes is clearly explained. The three most common and significant antioxidant enzymes include glutathione peroxidase, catalase, and superoxide dismutase. The four remaining antioxidant enzymes are glu- tathione reductase, thioredoxin reductase, heme oxygenase, and biliverdin reductase. People often get antioxidant enzymes from supplements or foods containing live enzymes. Foods containing live antioxidant enzymes include algae, yeast, and sprouts. Also, raw vegetables, barley grass, and wheatgrass contain high levels of antioxidant enzymes.

\section{Conflict of Interest}

The author declare that there is no any conflict of interest.

\section{Bibliography}

1. Kurutas EB. "The importance of antioxidants which play the role in cellular response against oxidative/nitrosative stress: current state". Nutrition Journal 15.71 (2016): 1-22.

2. Singh K., et al. "Antioxidants as precision weapons in war against cancer chemotherapy induced toxicity - Exploring the armoury of obscurity". Saudi Pharmaceutical Journal 26.2 (2018): 177-190.

3. Ali SS., et al. "Indian medicinal herbs as sources of antioxidants". Food Research International 41.1 (2008): 1-15.

4. Evans P and Halliwell B. "Free radicals and hearing: Cause, consequence, and criteria". Annals of the New York Academy of Sciences 884 (1999): 19-40.

5. Neta P., et al. "Rate constants for reactions of peroxyl radicals in fluid solutions". Journal of Physical and Chemical Reference Data 19 (1990): 413-513.

6. Hawkins CL and Davies MJ. Generation and propagation of radical reactions on proteins". Biochimica et Biophysica Acta 1504.2-3 (2001): 196-219.

7. Haber F and Weiss J. "The catalytic decomposition of hydrogen peroxide by iron salts". Proceedings of the Royal Society 147 (1934): 332-351.

8. Lloyd RV., et al. "The origin of the hydroxyl radical oxygen in the Fenton reaction". Free Radical Biology and Medicine 22.5 (1997): 885-888.

9. Collin F. "Chemical Basis of Reactive Oxygen Species Reactivity and Involvement in Neurodegenerative Diseases". International Journal of Molecular Sciences 20.10 (2019): 2407.

10. Kurutas EB., et al. "The effects of oxidative stress in urinary tract infection". Mediators of Inflammation 4 (2005): 242-244.

11. Valko M., et al. "Free radicals and antioxidants in normal physiological functions and human disease". The International Journal of Biochemistry and Cell Biology 39.1 (2007): 44-84.

12. Barbacanne MA., et al. "Detection of superoxide anion released extracellularly by endothelial cells using cytochrome $\mathrm{c}$ reduction, ESR, fluorescence and lucigenin-enhanced chemiluminescence techniques". Free Radical Biology and Medicine 29.5 (2000): 388-396. 
13. Masters CJ. "Cellular signalling: the role of the peroxisome". Cell Signal 8.3 (1996): 197-208.

14. Dröge W. "Free radicals in the physiological control of cell function”. Physiology Review 82.1 (2002): 47-95.

15. Valko M., et al. "Free radicals, metals and antioxidants in oxidative stress-induced cancer". Chemico-Biological Interactions 160.1 (2006): 1-40.

16. Haida $\mathrm{Z}$ and Hakiman M. "A comprehensive review on the determination of enzymatic assay and nonenzymatic antioxidant activities". Food Science and Nutrition 7.5 (2019): 15551563.

17. Mangge H., et al. "Antioxidants, inflammation and cardiovascular disease". World Journal of Cardiology 6.6 (2014): 462-477.

18. Ighodaro OM and Akinloye OA. "First line defence antioxidants-superoxide dismutase (SOD), catalase (CAT) and glutathione peroxidase (GPX): Their fundamental role in the entire antioxidant defence grid". Alexandria Journal of Medicine 54.4 (2018): 287-293.

19. Younus H. "Therapeutic potentials of superoxide dismutase". International Journal of Health Sciences 12.3 (2018): 88-93.

20. Yasui K and Baba A. "Therapeutic potential of superoxide dismutase (SOD) for resolution of inflammation". Inflammation Research 55.9 (2006): 359-363.

21. Inal ME., et al. "Antioxidant enzyme activities and malondialdehyde levels related to aging". Clinica Chimica Acta 305.1-2 (2001): 75-80.

22. Mulgund A., et al. The Role of Oxidative Stress in Endometriosis, Handbook of Fertility, Nutrition, Diet, Lifestyle and Reproductive Health (2015): 273-281.

23. Sharma I and Ahmad P. "Catalase: A Versatile Antioxidant in Plants". In Oxidative Damage to Plants, Antioxidant Networks and Signaling (2014): 131-148.

24. Bratovcic A and Saric E. "Biogenic Elements as Cofactors in Enzymes and Their Amount in the Chia Seed”. In: Karabegović I. (eds) New Technologies, Development and Application II. NT 2019. Lecture Notes in Networks and Systems. Springer, Cham (2020).

25. Bratovcic A and Saric E. "Determination of essential nutrients and cadmium in the white quinoa and amaranth seeds". Croatian Journal of Food Science and Technology 11.1 (2019): 1351390.

26. Eintemiller RR and Lee J. "Vitamin E: Food chemistry, composition and analysis". Marcel Dekker, New York (2004).

27. Traber MG and Atkinson J. "Vitamin E, antioxidant and nothing more”. Free Radical Biology and Medicine 43.1 (2007): 4-15.
28. Buettner GR. "The pecking order of free radicals and antioxidants: lipid peroxidation, alpha-tocopherol, and ascorbate". Archives of Biochemistry and Biophysics 300.2 (1993): 535-543.

29. Neuzil J., et al. "Alpha-tocopheryl hydroquinone is an efficient multifunctional inhibitor of radical-initiated oxidation of low density lipoprotein lipids". Proceedings of the National Academy of Sciences of the United States of America 94.15 (1997): 7885-7890.

30. Traber and Stevens. "Vitamins C and E: Beneficial effects from a mechanistic perspective". Free Radical Biology and Medicine 51.5 (2011): 1000-1013.

31. Kancheva VD., et al. "Bio-antioxidants-A chemical base of their antioxidant activity and beneficial effect on human health". Current Medicinal Chemistry 20.37 (2013): 4784-4805.

32. Griffiths K., et al. "Food Antioxidants and Their Anti-Inflammatory Properties: A Potential Role in Cardiovascular Diseases and Cancer Prevention". Diseases 4.3 (2016): 28.

33. Franco R., et al. "Chemical rules on the assessment of antioxidant potential in food and food additives aimed at reducing oxidative stress and neurodegeneration". Food Chemistry 235 (2017): 318-323.

34. Jurikova T., et al. "Fruits of black chokeberry aronia melanocarpa in the prevention of chronic diseases". Molecules 22.6 (2017): 944.

35. Bratovčić A., et al. "Determination of physical-chemical properties and stability of orange juice at room temperature". Sixth International Scientific Conference, June 5th - World Environment Day 6.6 (2018): 76-88.

36. Morita M., et al. "Antioxidant action of fermented grain food supplement: Scavenging of peroxyl radicals and inhibition of plasma lipid oxidation induced by multiple oxidants". Food Chemistry 237 (2017): 574-580.

37. Ayala A., et al. "Lipid peroxidation: Production, metabolism, and signaling mechanisms of malondialdehyde and 4-hydroxy-2-nonenal". Oxidative Medicine and Cellular Longevity (2014): 1-33.

38. Conti V., et al. "Antioxidant Supplementation in the Treatment of Aging-Associated Diseases". Frontiers in Pharmacology 7 (2016): 1-11.

39. Wells WW and Xu DP. "Dehydroascorbate reduction". Journal of Bioenergetics and Biomembranes 26 (1994): 369-377.

40. Cardenas E and Ghosh R. "Vitamin E: a dark horse at the crossroad of cancer management". Biochemistry and Pharmacology 86.7 (2013): 845-852.

41. Harikumar KB and Aggarwal BB. "Resveratrol: a multitargeted agent for age-associated chronic diseases". Cell Cycle 7 (2008): 1020-1035. 
42. Bhullar KS., et al. "Curcumin and its carbocyclic analogs: structure-activity in relation to antioxidant and selected biological properties". Molecules 18 (2013): 5389-5404.

43. Brondino N., et al. "Curcumin as a therapeutic agent in dementia: amini systematic review of human studies". The Scientific World Journal (2014): 174282.

44. Waterman E and Lockwood B. "Active components and clinical applications of olive oil". Alternative Medicine Review 12 (2007): 331-342.

45. Notomista E., et al. "Tuning the specificity of the recombinant multicomponent tolueneo-xylene monooxygenase from Pseudomonas sp. strain OX1 for the biosynthesis of tyrosol from 2-phenylethanol". Applied and Environmental Microbiology 77 (2011): 5428-5437.

46. Bulotta S., et al. "Beneficial effects of the olive oil phenolic components oleuropein and hydroxytyrosol: focus on protection against cardiovascular and metabolic diseases". Journal of Translational Medicine 12 (2014): 219.

47. Bhagavan HN and Chopra RK. "Plasma coenzyme Q10 response to oral ingestion of coenzyme Q10 formulations". Mitochondrion 7 (2007): S78-S88.

48. Forsmark-Andrée P., et al. "Endogenous ubiquinol prevents protein modification accompanying lipid peroxidation in beef heart submitochondrial particles". Free Radical Biology and Medicine 19 (1995): 749-757.

49. Bentinger M., et al. "The antioxidant role of coenzyme Q". Mitochondrion 7 (2007): S41-S50.

\section{Assets from publication with us}

- Prompt Acknowledgement after receiving the article

- Thorough Double blinded peer review

- Rapid Publication

- Issue of Publication Certificate

- High visibility of your Published work

Website: https://www.actascientific.com/

Submit Article: https://www.actascientific.com/submission.php Email us: editor@actascientific.com

Contact us: +91 9182824667 